\title{
Enseignement de la littérature : aspects français d'une crise mondiale
}

Emmanuel Fraisse ${ }^{a}$

\begin{abstract}
Résumé
Pour avoir fait des études littéraires un outil décisif de son projet éducatif et national, la France, avec un certain retard par rapport à d'autres pays occidentaux, est aujourd'hui conduite à réviser la place de la littérature dans l'ensemble de son dispositif de formation. Les réponses qu'elle donne à la crise de l'enseignement de la littérature et de son rayonnement social different selon les degrés d'enseignement. Dans l'enseignement scolaire obligatoire (jusqu'à 16 ans), on observe une prise en compte de la notion de compétence; l'enseignement secondaire long (16-18 ans) a connu une rénovation partielle mais réelle au plan des objets et des méthodes au cours de ces dernières années. En revanche, l'université apparait plus conservatrice et la marche vers l'invention $d$ ' "humanités contemporaines » est entravée par un enchevêtrement de facteurs au premier rang desquels la peur du nouveau et le sentiment de la crise. Or, en France comme dans le reste du monde, c'est la capacité à dessiner sans préjugés le visage des futures études culturelles qui est en jeu, et à travers elle le développement de nouveaux objets, de nouveaux exercices, de nouvelles méthodes et d'une véritable interdisciplinarité.
\end{abstract}

Mots-clés: littérature ; enseignement ; études culturelles

a Professor e diretor de relações internacionais da Université de Paris 3, Sorbonne Nouvelle. fraisse.emma@ orange.fr 
1 «Une Nation, c'est une âme, un principe spirituel. Deux choses qui à vrai dire n'en font qu'une - constituent cette âme, ce principe spirituel. L'un est dans le passé, l'autre est dans le présent. L'une est la possession commune d'un riche legs de souvenirs ; l'autre est le consentement mutuel, le désir de vivre ensemble, la volonté de continuer à faire valoir l'héritage qu'on a reçu indivis ». Ernest Renan, Qu'est-ce qu'une nation? Conférence faite à la Sorbonne le 11 mars 1882, début du ch. 3. http://www. b m lis i e u x.com / archives/nation01.htm

2 Voir Observatoire des inégalités, http:// www.inegalites.fr/ spip.php?article881 La scolarisation des enfants dans le monde", 2 septembre 2013.

3 Voir Marcel Gauchet «Contre l'idéologie de la compétence, l'école doit apprendre à penser », Le Monde, 02/09/2011.

${ }^{4}$ Voir http://www. education.gouv.fr/ cid75345/1-evolutiond e s - a c qui s - d e s eleves-de-15-ans-encomprehension-de-1ecrit.html

${ }^{5}$ Dans un contexte de questionnement finalement très stable, voir l'analyse très nuancée de Elisabeth Bautier, Jacques Crinon, Patrick Rayou et Jean-Yves Rochex, «Performances en littéracie, modes de faire et univers mobilisés par les élèves : analyses secondaires de l'enquête PISA 2000 ", Revue française de pédagogie, $\mathrm{n}^{\circ} 157$, octobre-décembre 2006, p. 85-101.
Parce qu'elle a longtemps érigé son modèle humaniste en idéal d'éducation, fait des études littéraires son principal outil de formation des élites et le vecteur privilégié de l'établissement d'un corpus commun de référence établissant des valeurs explicites et partagées, l'école française d'aujourd'hui subit avec plus de force que d'autres systèmes éducatifs nationaux la crise durable et désormais ancienne que connaît l'enseignement de la littérature. Cette crise est d'autant plus sensible en France que l'idée même de littérature y a été affirmée de manière indissoluble comme nationale ${ }^{1}$ et universelle : si la France, pensait-on, avait bien une place à part au plan culturel, c'était pour avoir su actualiser et exprimer dans sa langue les messages susceptibles de mobiliser et éclairer l'humanité dans son ensemble. Et à travers un paradoxe ressenti par beaucoup comme une évidence, la particularité française résidait donc dans une large mesure dans l'universalité de sa littérature et le « génie " propre de sa langue.

Même si de nombreuses inégalités demeurent aujourd'hui dans le domaine de l'éducation dans le monde (ainsi seuls 29 $\%$ des africains fréquentent le secondaire court contre $92 \%$ dans les pays occidentaux) et si l'on doit prendre en compte une certaine surestimation des chiffres des populations scolarisées à l'échelle du globe, une des caractéristiques de la phase actuelle de la mondialisation réside dans l'extension sans précédent de la scolarisation, $63 \%$ des élèves dans le monde poursuivant des études jusqu'à 16 ans $^{2}$. Or parallèlement à cette extension des effectifs scolarisés à une échelle jusqu'ici inconnue et naguère inconcevable, on observe des changements assez radicaux dans les curricula et notamment un primat accordé aux compétences réelles sur les connaissances théoriques, volontiers considérées comme abstraites ${ }^{3}$. Pour une part cette évolution s'inscrit dans des objectifs d'efficacité, d'utilité et d'adaptation aux réalités économiques et sociales de notre temps. À cet égard, les mesures de la compréhension de l'écrit chez les élèves de 15 ans opérées depuis 2000 par l'OCDE dans le cadre du Programme for international Student Assessment (PISA), médiocres pour la France d'autant qu'ils font apparaître des inégalités importantes entre bons et mauvais élèves ${ }^{4}$, sont massivement liées à des questionnements relatifs à des écrits fonctionnels et très secondairement à des écrits relevant de la fiction ou de la littérature proprement dite ${ }^{5}$. 


\section{Fin des humanités d'hier}

Dans ces conditions, on ne s'étonnera pas trop de constater que la crise des approches humanistes soit lisible à l'échelle du monde, et tout particulièrement dans les segments d'enseignement où elle était déterminante, notamment dans la formation des élites. Hier le latin fixait une barrière décisive : ce rôle est aujourd'hui dévolu à la culture scientifique et mathématique. Or cette question du latin ${ }^{6}$ (et accessoirement du grec), fondamentale dans les difficultés de l'apparition progressive d'un enseignement moderne en France vers $1900^{7}$ était, avec bien des variations, commune à tous les systèmes de formation des élites occidentales qui visaient largement à former des orateurs et des professeurs plus que des hommes d'affaires et des ingénieurs ${ }^{8}$. C'est ainsi que, dans ses mémoires de jeunesse, Winston Churchill, qui fut un très mauvais élève, étalait sa supériorité paradoxale sur ses camarades plus avancés que lui en Grec et en Latin puisque, à cause même de

${ }^{6}$ Voir notamment Philippe Cibois, «La question du latin, des critiques du XVIII au revival du XIX" ${ }^{\mathrm{e}}$, L'information littéraire, $\mathrm{n}^{\circ} 52,2000 / 1$, p. 7-28. Voir également Raoul Frary, La question $d u$ latin, Paris, Léopold Cerf, 1885. Pour une synthèse de la question des lettres "modernes" dans l'enseignement supérieur depuis 1945 voir Yves Chevrel, «Les lettres et la formation des professeurs de français ", L'information littéraire, 53, 2003/3, p. 3-10.

7 Violaine HoudartMerot, La culture littéraire au lycée depuis 1880, Rennes/ Paris: Presses universitaires de Rennes/Adapt éditions, 1998, 274 p.

8 V o i r A n d r é Chervel, Histoire de l'enseignement du français du XVII ${ }^{\mathrm{e}}$ au $\mathrm{XX}^{\mathrm{e}}$ siècle, Paris : Retz, 2006.

${ }^{9}$ Winston Churchill, My Early Life, 18741904, [1930], London: Oldhams, 1965, p. 18-19. son retard et de sa fermeture aux apprentissages scolaires, il avait eu l'occasion de faire sien l'anglais et d'incorporer sa musique, son rythme et « la structure essentielle de la phrase anglaise ordinaire » :

[B]y being so long in the lowest form I gained an immense advantage over the cleverer boys. They all went on to learn Latin and Greek and splendid things like that. But I was taught English. We were considered such dunces that we could learn only English. Mr. Somervell - a most delightful man, to whom my debt is great - was charged with the duty of teaching the stupidest boys the most disregarded thing - namely, to write mere English. He knew how to do it. He taught it as no one else has ever taught it. Not only did we learn English parsing thoroughly, but we also practised continually English analysis... Thus I got into my bones the essential structure of the ordinary British sentence - which is a noble thing. And when in after years my schoolfellows who had won prizes and distinction for writing such beautiful Latin poetry and pithy Greek epigrams had to come down again to common English, to earn their living or make their way, I did not feel myself at any disadvantage. Naturally I am biased in favour of boys learning English. I would make them all learn English: and then I would let the clever ones learn Latin as an honour, and Greek as a treat. But the only thing I would whip them for would be not knowing English. I would whip them hard for that. ${ }^{9}$ 
10 AuxÉtats-Unis, cen'est que progressivement que l'Anglais et $l^{\prime}$ « English Composition » apparaissent comme objets d'enseignement universitaire : l'anglais n'est inscrit à l'examen d'entrée de Harvard qu'en 1873-1874 seulement et la vogue de l' " English Composition » (opposée aux approches philologiques) se fait sentir à partir des années 1890 sous l'influence de Barrett Wendell. Voir Anne-Marie Petitjean, La littérature sur le métier: Étude comparée des pratiques créatives d'écriture littéraires dans les universités, en France, aux États-Unis et au Québec, thèse pour le doctorat, Université de CergyPontoise, 2013, p. 147-150.

${ }^{11}$ En France, 1e baccalauréat général et ses 3 filières - Sciences, Sciences économiques et sociales, Lettres représente la moitié des effectifs concernés (36 $\%$ de la classe d'âge), les autres baccalauréats étant technologiques ou professionnels.

12 Voir http://insee. $\mathrm{fr} / \mathrm{fr} /$ themes/tableau. a sp?reg_id=0 \& ref id=NATC $\bar{C}$ F07106

13 La même perte d'attractivité est reconnue aux États-Unis par la Modern Language Association qui, dans son livre blanc de 2009 souligne l'accélération de ce phénomène au cours des quinze dernières années : "We know that today's students are less likely to choose language and literary study as majors than they were thirty-five or even fifteen years ago, and we wanted to explore ways to strengthen majors in our fields and attract new generations of students to what has been the traditional core of liberal study." Modern Language Association, Report to the Teagle Foundation on the Undergraduate Major in Language and Literature, Web publication, February 2009. http://www.mla. org/pdf/2008_mla_ whitepaper.pdf
Non seulement les langues anciennes, jadis au cœur du dispositif d'enseignement des élites, ont donc pratiquement disparu des programmes secondaires et universitaires français, mais c'est désormais la notion même de littérature comme objet d'enseignement qui est, sinon remise en cause, du moins considérée comme problématique. Là encore, une telle évolution n'a rien de spécifiquement français : tous les systèmes d'enseignement des pays occidentaux ont suivi, et avec plus de rapidité encore, un chemin analogue ${ }^{10}$. Mais compte tenu des spécificités françaises et du rôle des enseignements littéraires dans la psychè collective de ce pays, le choc est particulièrement rude.

Premier signe de ce choc, la chute progressive, régulière et apparemment inexorable de la filière littéraire du baccalauréat. Alors que plus d'un tiers des élèves présentant le bac (tous types de bac confondus ${ }^{11}$ ) choisissait cette filière en 1968, ils étaient encore 18,5 \% en 1994 pour n'être que 8\% aujourd'hui. De surcroît on relèvera le très haut taux de féminisation de cette filière : $80 \%$ contre $60 \%$ en Sciences économiques et sociales et $45 \%$ en Sciences ${ }^{12}$. D'une manière générale ces déterminations, visibles dans l'enseignement secondaire long se voient renforcées au plan universitaire : les effectifs des étudiants inscrits dans les filières littéraires, et tout particulièrement en littérature, n'ont cessé de reculer depuis 40 ans $^{13}$.

Second signe de crise, l'absence de consensus sur les contenus et méthodes des études littéraires, tant dans l'enseignement secondaire que dans l'enseignement universitaire malgré un effort considérable et continu de 
${ }_{14}$ De manière assez curieuse l'ordre entre les deux termes ne semble pas stabilisé au long de la rédaction du texte officiel qui fait donc alterner « compétences et connaissances » et "connaissances et compétences ».

${ }^{15}$ http://eduscol. education.fr/pid23199/ socle-commun.html

${ }^{16} \mathrm{~L}^{\prime}$ e $x$ p r e s s i o $n$ archétypale de cette division par siècle et de la tendance à réduire l'étude de la littérature à $l^{\prime}$ histoire littéraire proprement dite est à rechercher dans les manuels de Lagarde et Michard qui, depuis la fin de la Seconde guerre mondiale, continuent à servir de référence sinon aux élèves du moins à de nombreux professeurs et aux étudiants se préparant au professorat. André Lagarde et Laurent Michard, Textes et littérature, Les grands auteurs français $d u$ programme, Paris, Bordas, t. 1, Moyen Âge, 1948 ; t. 2 , XVI ${ }^{\mathrm{e}}$ siècle, 1949 ; t. 3, XVII ${ }^{\mathrm{e}}$ siècle, 1951 ; t. 4, XVIII ${ }^{\mathrm{e}}$ siècle 1953 ; t. 5, XIX ${ }^{\mathrm{e}}$ siècle, 1955 ; t. $6, X^{e}$ siècle, 1962.

${ }^{17}$ ht t p://edus col. education.fr/pid24316/ programmes-secondegenerale-technologique. html définition et de renouvellement des programmes dans l'enseignement scolaire. Cet effort est lisible notamment à travers l'adoption d'un "Socle commun de compétences et de connaissances ${ }^{14}$ » concernant tous les élèves au long de la scolarité jusqu'à 16 ans et adopté solennellement par le Parlement en 2005 :

Le socle commun est le ciment de la nation : il s'agit d'un ensemble de valeurs, de savoirs, de langages et de pratiques dont l'acquisition repose sur la mobilisation de l'école et qui suppose, de la part des élèves, des efforts et de la persévérance $^{15}$.

Déclinant les objectifs pédagogiques en «connaissances », « capacités » et « attitudes », le principe même du socle établit la recherche d'un compromis nouveau pour la tradition française si centrée sur les savoirs et finalement bien méprisante des savoir-faire. Reste que ce compromis n'entre que très progressivement dans les pratiques éducatives comme dans les opinions des enseignants et des parents d'élèves.

\section{Refonder les humanités}

Le même effort d'aggiornamento est également lisible dans la réécriture des programmes littéraires de l'enseignement secondaire long (lycée) pour les élèves de 16 à 18 ans en 2010 et 2011. On doit en effet relever un parti-pris de désenclavement des approches littéraires traditionnelles par le biais de l'ouverture à des problématiques liant "littérature et société » et une certaine rupture avec l'hégémonie de l'histoire littéraire traditionnelle et la division par siècles ${ }^{16}$ qui cède partiellement la place à une entrée par grands genres littéraires et vise à un équilibre entre "l'étude de la littérature dans son contexte historique et culturel et l'analyse des grands genres littéraires ${ }^{17}$ ». De même, on peut observer un effort d'ouverture vers le cinéma et l'image (notamment pour les littéraires de la classe terminale) et une volonté d'approches pluridisciplinaires concernant les arts et l'histoire. Il n'en demeure pas moins que le système de référence de la littérature reste très lent 
${ }^{18}$ Voir Violaine Houdart-Merot et Christine Mongenot (dir.), Pratiques d'écriture littéraire à l'université, Paris: Honoré Champion, coll." Didactique des lettres et des cultures », 2013.

19 Pour les chiffres 2012-2013, en recul de $2,8 \%$ en lettres et sciences du langage, voir http://cache.media. enseignementsuprecherche.gouv.fr/ file/2013/83/1/NI MESR_13_11_286831. pdf dans ses évolutions, d'autant que les exercices mis en œuvre par les élèves eux-mêmes renvoient à des cadres eux-mêmes très stables. Alors qu'aux épreuves écrites du baccalauréat (qui pour le français dans toutes les sections concerne les élèves de la classe de première), trois exercices peuvent être choisis : commentaire, dissertation ou " écriture d'invention ", cette dernière, tard venue, fait figure de suspecte auprès de la plupart des professeurs qui hésitent à la recommander et à la faire travailler d'autant qu'elle peut offrir des difficultés d'appréciation, de correction et de notation. Quant aux élèves ils sont dans l'ensemble réticents face à cet exercice, compte tenu de l'idée qu'ils se font de la discipline «français » et de leurs stratégies de réussite à l'examen.

Des réticences plus fortes encore se font sentir à l'université face aux formes d'écriture créative qui pourraient être directement intégrées dans les cursus littéraires : pour l'essentiel les études littéraires sont perçues comme exercice de critique érudite et distante, pas d'imitation et encore moins de production et de création ${ }^{18}$. Or c'est justement à l'université, lieu décisif de la formation des futurs professeurs, que la crise de la discipline, et son manque d'évolution se font le plus sentir.

Au-delà de la question de la prise en compte éventuelle des approches créatives, ces difficultés touchent massivement la définition ou l'extension des domaines d'études considérés comme le plus légitimes. Les effectifs sont en recul dans la plupart des établissements, et notamment les plus petits ${ }^{19}$. Les départements de Lettres (ou de « Lettres modernes » compte tenu de la quasi disparition des langues anciennes) expriment dans leur majorité de fortes préventions face à l'étude des littératures francophones, pourtant particulièrement reconnues, documentées, inventives et vivaces. Il en va de même des études de genre et de l'analyse de spécificités de la littérature féminine. Quant à l'étude des littératures d'enfance et de jeunesse, elles aussi très inventives et massivement présentes dans l'enseignement élémentaire, elles restent sauf exception à la portion congrue dans l'enseignement universitaire. Tant en ce qui concerne littératures francophones ou d'enfance, on assiste donc à un paradoxe lourd de menaces : des œuvres nombreuses et reconnues circulent, des travaux critiques de qualité sont publiés, de très nombreux jeunes chercheurs s'investissent dans ces domaines, mais ils manquent à la fois 
${ }^{20}$ Étudiantla ventilation des quelque 225 thèses littéraires soutenues dans les universités françaises en 2007 nous avions observé que plus d'une thèse sur deux concernait le $X X^{e}$ siècle. Conséquence de cette prédominance, les autres périodes historiques étaient très minoritaires : 27 pour le $\mathrm{XIX}^{\mathrm{e}}$ siècle, 12 pour le XVIII siècle, 11 pour le XVII ${ }^{e}$ siècle, 3 pour le $\mathrm{XVI}^{\mathrm{e}}$ et 5 pour le Moyenâge. On relevait cette même année 200750 entrées $(22 \%)$ touchant la francophonie ainsi qu'une présence notable d'études explicitement féminines (10 titres) ou liées à la jeunesse (7 titres). Emmanuel Fraisse, "L'interprétation des textes littéraires à l'université ", in Max Butlen et Violaine Houdart-Merot (dir.), Interpréter et transmettre la littérature aujourd'hui, Amiens, Encrage université, diffusion Les Belles lettres, 2009, p. 123-131.

${ }^{21}$ Voir Philippe Caron, Aux origines de la notion contemporaine de "Littérature". Le Lexique et la c on fig u ration idéologique des grands secteurs du savoir profane en langue française de 1680 à 1760 , Thèse pour le doctorat, Université de Nancy 2, 1987. d'encadrement et de débouchés alors que les domaines les plus légitimes et les plus fréquentés hier encore sont très largement désertés ${ }^{20}$.

Plus largement, et au plan structurel, c'est l'idée même de départements universitaires ou de structures d'enseignement et de recherche voués à la littérature française stricto sensu qui mérite d'être reconsidérée. Si on peut penser que les formations consacrées exclusivement à tel ou tel domaine d'études (" Studies ») présentent la faiblesse de leur liaison directe à la mode et de leur relative étroitesse, il en va bien autrement des « Cultural Studies» dont le champ d'application est très vaste et interdisciplinaire par nature. Il s'agit en fait de mobiliser des types d'organisation de l'enseignement et de la recherche - les deux gagnent en effet à être liés sans pour autant être confondus à tout moment - susceptibles de nous faire mieux comprendre la complexité et l'interaction des phénomènes culturels et de participer ainsi à un renouvellement de l'idée même de littérature.

On sait en effet que la notion de "littérature » a considérablement évolué au cours du temps ${ }^{21}$ et qu'elle connaît également des variations dans l'espace selon les différentes aires culturelles : dans quelle mesure l'écriture de l'histoire relève-t-elle de la littérature, ici ou là, hier ou aujourd'hui ? L'idée de "littérature » que nous partageons plus ou moins aujourd'hui, sans jamais vraiment la définir autrement que par des exemples et des listes, est donc évidemment conduite à se transformer à l'avenir. Quant aux études littéraires, elles ont connu également des redéfinitions constantes. Il importe désormais que nous soyons capables, au plan de la recherche comme de l'enseignement, de proposer une approche infiniment plus ouverte des objets et des méthodes que l'ensemble des enseignants et chercheurs en "études culturelles » (et pas seulement les littéraires) puissent partager et approfondir. Ces domaines d'étude peuvent, sans dommage, prendre un nom fort ancien, respectable et ouvert à tous les possibles : celui d'humanités. Humanités, ces connaissances profanes qui permettent de mieux connaître l'homme et nous rendent plus humains : litterae humaniores comme on l'écrivait au Moyen Âge. Et là, l'affaire n'est évidemment pas française, mais mondiale. 


\section{Resumo}

\section{Ensino de literatura: aspectos franceses de uma crise global}

Como a França fez dos estudos literários um instrumento decisivo do seu projeto educativo e nacional, hoje, com um certo atraso em relação a outros países ocidentais, ela se vê levada a rever o lugar da literatura no conjunto de seu dispositivo de formação. As respostas que ela dá à crise do ensino da literatura e de seu alcance social são diferentes, segundo o nível de ensino. No ensino escolar obrigatório (até 16 anos), observa-se uma tomada de consciência da noção de competência; o ensino secundário longo (16-18 anos) sofreu uma renovação parcial, mas real, em relação aos objetos e métodos, durante os últimos anos. Por outro lado, a universidade é mais conservadora, e o avanço para a invenção das "humanidades contemporâneas" é travado por um conjunto de fatores, dos quais o primeiro seria o medo da novidade e o sentimento da crise. Ora, na França, como no resto do mundo, o que está em jogo é a capacidade de enfrentar, sem preconceitos, a imagem dos futuros estudos culturais, e, através dela, o desenvolvimento de novos objetos, de novos exercícios, de novos métodos e de uma verdadeira interdisciplinaridade.

Palavras-chave: literatura; ensino; estudos culturais. 


\begin{abstract}
Teaching literature: French aspects of a global crisis

For making literary studies a decisive tool of its education and national project, France, with some delay compared to other western countries, is now taken to revise the role of literature in all its forming device. The answers it gives to the crisis in the teaching of literature and its social influence differ across levels of education. In compulsory school education (up to 16), there is a consideration of the concept of competence; upper secondary education (16-18 years) had a partial but real renewal in terms of objects and methods in recent years. However, the university appears more conservative and walking towards the invention of "contemporary classics" is hampered by a tangle of factors chief among them the fear of the new and the feeling of the crisis. However, in France as in the rest of the world, it is the ability to draw unprejudiced face future cultural studies is at stake, and through it the development of new objects, new exercises, new methods and a true interdisciplinarity.
\end{abstract}

Keywords: literature; teaching; cultural studies 